\title{
The diagonal mapping in mixed norm spaces
}

\author{
by \\ Guangbin Ren (Hefei and Aveiro) and Jihuai Shi (Hefei)
}

\begin{abstract}
For any holomorphic function $F$ in the unit polydisc $U^{n}$ of $\mathbb{C}^{n}$, we consider its restriction to the diagonal, i.e., the function in the unit disc $U$ of $\mathbb{C}$ defined by $\mathcal{D} F(z)=F(z, \ldots, z)$, and prove that the diagonal mapping $\mathcal{D}$ maps the mixed norm space $H^{p, q, \alpha}\left(U^{n}\right)$ of the polydisc onto the mixed norm space $H^{p, q,|\alpha|+(p / q+1)(n-1)}(U)$ of the unit disc for any $0<p<\infty$ and $0<q \leq \infty$.
\end{abstract}

1. Introduction. Let $U^{n}$ be the polydisc in $\mathbb{C}^{n}$ and $T^{n}$ be its Shilov boundary (see [Ru1]). Denote by $d m_{n}$ the normalized volume measure in $U^{n}$, and by $d \sigma_{n}$ the normalized surface measure on $T^{n}$. For any Lebesgue measurable function $f$ in $U^{n}$, we define

$$
M_{q}(r, f)=\left(\int_{T^{n}}|f(r \zeta)|^{q} d \sigma_{n}(\zeta)\right)^{1 / q}
$$

where $0<q<\infty$ and $r \zeta=\left(r_{1} \zeta_{1}, \ldots, r_{n} \zeta_{n}\right)$. When $q=\infty$, as usual, we define $M_{\infty}(r, f)$ to be the essential supremum of $|f(r \zeta)|$ over $\zeta \in T^{n}$. If $0<p<\infty, 0<q \leq \infty$, and $\alpha=\left(\alpha_{1}, \ldots, \alpha_{n}\right), \alpha_{j}>-1, \forall j=1, \ldots, n$, let

$$
\|f\|_{p, q, \alpha}^{p}=\int_{I^{n}} \prod_{j=1}^{n}\left(1-r_{j}^{2}\right)^{\alpha_{j}} M_{q}^{p}(r, f) d r,
$$

where $I^{n}=[0,1)^{n}$ and $d r=d r_{1} \cdots d r_{n}$. The mixed norm space $L^{p, q, \alpha}\left(U^{n}\right)$ is then defined to be the space of functions $f$ in $U^{n}$ such that $\|f\|_{p, q, \alpha}<\infty$, and the holomorphic mixed norm space $H^{p, q, \alpha}\left(U^{n}\right)$ is its subspace consisting of holomorphic functions. The mixed norm spaces have been studied extensively; see, for example, [BP], [AJ], [J], [L], [Sh2], [Pa] and [SR].

The main purpose of this article is to consider the action of the diagonal mapping on mixed norm spaces on $U^{n}$.

2000 Mathematics Subject Classification: 32A37, 47B38.

Key words and phrases: diagonal mapping, mixed norm spaces, weighted Bergman projection.

The first author was supported in part by the NNSF of China (\#10001030) and a Post-doctoral Fellowship of the University of Aveiro, UI\&D "Matemática e Aplicações". 
To each holomorphic function $F$ in the unit polydisc $U^{n}$ of $\mathbb{C}^{n}$, we associate a function $\mathcal{D} F$, defined on the unit disc $U$ of $\mathbb{C}$ by

$$
\mathcal{D} F(z)=F(z, \ldots, z) .
$$

The operator $\mathcal{D}$ is called the diagonal mapping. In his book [Ru1], Rudin suggested the study of this mapping. Afterwards, the diagonal mapping has been completely investigated in the Hardy spaces and Bergman spaces; see [Ru1], [HO], [DS], [Sha], [MR], [Sh], [Djs], and [RL]. For instance, Shapiro [Sha] and Shamoian $[\mathrm{Sh}]$ proved that

$$
\mathcal{D} H^{p, p, \alpha}\left(U^{n}\right)=H^{p, p,|\alpha|+2 n-2}(U)
$$

for any $0<p<\infty$ and $\alpha_{j}>-1, \forall j=1, \ldots, n$, where $|\alpha|=\alpha_{1}+\cdots+\alpha_{n}$.

In view of (1.4), the interesting phenomenon in weighted Bergman spaces is that for any given weight $\alpha$, the resulting weight $|\alpha|+2 n-2$ is independent of $p$. But this fails in mixed norm spaces, i.e.,

$$
\mathcal{D} H^{p, q, \alpha}\left(U^{n}\right) \neq H^{p, q,|\alpha|+2 n-2}(U) .
$$

In fact, taking $f\left(z_{1}, z_{2}\right)=\left(1-z_{1}\right)^{-\beta_{1}}\left(1-z_{2}\right)^{-\beta_{2}}$ with $\beta_{i}=\left(1+\alpha_{i}\right) / 2+4 / 5$ for $i=1,2$, one can easily verify that $f \in H^{2,1, \alpha}\left(U^{2}\right)$ but $\mathcal{D} f \notin H^{2,1,|\alpha|+2}(U)$.

Our main result is the following theorem.

Theorem 1.1. Let $0<p<\infty, 0<q \leq \infty$ and let $\alpha=\left(\alpha_{1}, \ldots, \alpha_{n}\right)$, $\alpha_{j}>-1, \forall j=1, \ldots, n$. Then

$$
\mathcal{D} H^{p, q, \alpha}\left(U^{n}\right)=H^{p, q,|\alpha|+(p / q+1)(n-1)}(U) .
$$

Theorem 1.1 also shows that, by the closed graph theorem, the composition operator $C_{\Phi}$ defined by

$$
C_{\Phi} F=F \circ \Phi,
$$

where $\Phi(z)=(z, \ldots, z)$ for any $z \in U$, is bounded from $H^{p, q, \alpha}\left(U^{n}\right)$ onto $H^{p, q,|\alpha|+(p / q+1)(n-1)}(U)$. For the theory of composition operators, we refer to $[\mathrm{CM}]$.

The paper is organized as follows. In the next section, we provide an integral representation for the diagonal mapping, given by the diagonalization of weighted Bergman operators of $U^{n}$. Similarly, we provide an integral representation for a right inverse operator of the diagonal mapping, given by the polarization of weighted Bergman operators of $U$. In the third section, we extend Hardy's inequalities ([HL], $[\mathrm{Fl}],[\mathrm{AB}])$ to higher dimensions, which is a key tool to proving the boundedness of integral operators in mixed norm spaces. In the fourth section, we show that the weighted Bergman projection $\mathcal{T}_{\beta}$, which is the orthogonal projection from $L^{2}\left(U^{n}, \prod_{j=1}^{n}\left(1-\left|u_{j}\right|^{2}\right)^{\beta_{j}} d m_{n}(u)\right)$ onto $H^{2,2, \beta}\left(U^{n}\right)$, induces a bounded operator from $L^{p, q, \alpha}\left(U^{n}\right)$ to $H^{p, q, \alpha}\left(U^{n}\right)$. 
2. Diagonalization and polarization. Set $\alpha=\left(\alpha_{1}, \ldots, \alpha_{n}\right)$ and $\beta=$ $\left(\beta, \ldots, \beta_{n}\right)$.

Hypothesis 2.1. Throughout the paper, we assume that

(i) $0<p<\infty$ and $0<q \leq \infty$;

(ii) $\alpha_{j}>-1, \quad \forall j=1, \ldots, n$;

(iii) $\beta_{j}>\frac{\alpha_{j}+1}{p}+\frac{1}{\min (q, 1)}-2, \quad \forall j=1, \ldots, n$.

By Hypothesis 2.1, it is clear that always $\beta_{j}>-1$. We remark that Hypothesis 2.1(iii) is required only to assure the validity of technical lemmas in our applications, i.e., Lemmas 3.2, 3.3 and 4.2 below.

For any $u \in U^{n}$ and $r \in I^{n}$, we write $u=\left(u_{1}, \ldots, u_{n}\right)$ and $r=$ $\left(r_{1}, \ldots, r_{n}\right)$. We shall often use the polar coordinates formula:

$$
\int_{U^{n}} \prod_{j=1}^{n}\left(1-\left|u_{j}\right|^{2}\right)^{\beta_{j}}|F(u)|^{p} d m_{n}(u)=2^{n} \int_{I^{n}} \prod_{j=1}^{n} r_{j}\left(1-r_{j}^{2}\right)^{\beta_{j}} M_{p}^{p}(r, F) d r
$$

for any measurable function $F$ in $U^{n}$. Further, if $F$ is holomorphic, then it is well known (see [Sh1]) that

$$
\int_{U^{n}} \prod_{j=1}^{n}\left(1-\left|u_{j}\right|^{2}\right)^{\beta_{j}}|F(u)|^{p} d m_{n}(u) \simeq \int_{I^{n}} \prod_{j=1}^{n}\left(1-r_{j}^{2}\right)^{\beta_{j}} M_{p}^{p}(r, F) d r,
$$

where $A \simeq B$ means $K^{-1} A \leq B \leq K A$. Here and afterwards, $K$ always denotes some positive absolute constant which may vary from line to line.

Our starting point is the weighted Bergman projection operator. For any function $F$ in $U^{n}$ and $v \in U^{n}$, we let

$$
\mathcal{T}_{\beta} F(v)=\prod_{j=1}^{n}\left(\beta_{j}+1\right) \int_{U^{n}} \frac{\prod_{j=1}^{n}\left(1-\left|u_{j}\right|^{2}\right)^{\beta_{j}}}{\prod_{j=1}^{n}\left(1-v_{j} \bar{u}_{j}\right)^{\beta_{j}+2}} F(u) d m_{n}(u) .
$$

It is well known that $\mathcal{T}_{\beta}$ is the orthogonal projection from the Hilbert space $L^{2}\left(U^{n}, \prod_{j=1}^{n}\left(1-\left|u_{j}\right|^{2}\right)^{\beta_{j}} d m_{n}(u)\right)$ onto its holomorphic Hilbert subspace, i.e. the weighted Bergman space $H^{2,2, \beta}\left(U^{n}\right)$.

We also consider the diagonalization and polarization of the Bergman projection. For any functions $F$ in $U^{n}$ and $f$ in $U$, we define functions $\mathcal{D}_{\beta} F$ in $U$ and $\mathcal{E}_{\beta} f$ in $U^{n}$ :

$$
\begin{aligned}
\mathcal{D}_{\beta} F(z) & =\prod_{j=1}^{n}\left(\beta_{j}+1\right) \int_{U^{n}} \frac{\prod_{j=1}^{n}\left(1-\left|u_{j}\right|^{2}\right)^{\beta_{j}}}{\prod_{j=1}^{n}\left(1-z \bar{u}_{j}\right)^{\beta_{j}+2}} F(u) d m_{n}(u), \\
\mathcal{E}_{\beta} f(u) & =(|\beta|+2 n-1) \int_{U} \frac{\left(1-|z|^{2}\right)^{|\beta|+2 n-2}}{\prod_{j=1}^{n}\left(1-u_{j} \bar{z}\right)^{\beta_{j}+2}} f(z) d m_{1}(z) .
\end{aligned}
$$


It is clear that $\mathcal{D}_{\beta}$ is the diagonalization of the weighted Bergman operator $\mathcal{T}_{\beta}$ of $U^{n}$, and $\mathcal{E}_{\beta}$ is the polarization of the weighted Bergman projection $\mathcal{T}_{|\beta|+2 n-2}$ of $U$. We shall see that, when restricted to holomorphic mixed norm spaces, $\mathcal{D}_{\beta}$ coincides with the diagonal mapping $\mathcal{D}$, while $\mathcal{E}_{\beta}$ plays the role of a right inverse of $\mathcal{D}$.

THEOREM 2.2. Under Hypothesis 2.1,

(i) $\mathcal{T}_{\beta} f=f$ for every $f \in H^{p, q, \alpha}\left(U^{n}\right)$;

(ii) $\mathcal{D}_{\beta}: H^{p, q, \alpha}\left(U^{n}\right) \rightarrow H^{p, q,|\alpha|+(p / q+1)(n-1)}(U)$ is bounded;

(iii) $\mathcal{E}_{\beta}: H^{p, q,|\alpha|+(p / q+1)(n-1)}(U) \rightarrow H^{p, q, \alpha}\left(U^{n}\right)$ is bounded.

Corollary 2.3. Under Hypothesis 2.1, for any $F \in H^{p, q, \alpha}\left(U^{n}\right)$, we have $\mathcal{D} F \in H^{p, q,|\alpha|+(p / q+1)(n-1)}(U)$ and

$$
\mathcal{D}=\left.\mathcal{D}_{\beta}\right|_{H^{p, q, \alpha}\left(U^{n}\right)} .
$$

Proof. From (1.3), Theorem 2.2(i), (2.1) and (2.2), we have

$$
\mathcal{D} F(z)=F(z, \ldots, z)=\mathcal{T}_{\beta} F(z, \ldots, z)=\mathcal{D}_{\beta} F(z) .
$$

Therefore Theorem 2.2(ii) shows that $\left.\mathcal{D} F \in H^{p, q,|\alpha|+(p / q+1)(n-1)}(U)\right)$.

COROLlary 2.4. Under Hypothesis 2.1, for $f \in H^{p, q,|\alpha|+(p / q+1)(n-1)}(U)$, we have $\mathcal{E}_{\beta} f \in H^{p, q, \alpha}\left(U^{n}\right)$ and

$$
\mathcal{D}\left(\mathcal{E}_{\beta} f\right)=f .
$$

Proof. Let $\beta$ be a multi-index satisfying Hypothesis 2.1. For any $f \in$ $H^{p, q,|\alpha|+(p / q+1)(n-1)}(U)$, Theorem 2.2(iii) shows that $\mathcal{E}_{\beta} f \in H^{p, q, \alpha}\left(U^{n}\right)$.

Let $A\left(U^{n}\right)$ be the space of functions holomorphic in $U^{n}$ and continuous in the closure of $U^{n}$. When $n=1$, it is known [Sh2 (I), Proposition 2.3] that $A(U)$ is dense in $H^{p, q, \gamma}(U)$ for any $\gamma>-1$. From (2.4) and the boundedness of $\mathcal{D}_{\beta}$ and $\mathcal{E}_{\beta}$, as shown in Theorem 2.2(ii), (iii), we need only show that

$$
\mathcal{D}_{\beta}\left(\mathcal{E}_{\beta} f\right)=f, \quad \forall f \in A(U) .
$$

Fix $w \in U$ and define

$$
h_{w}(u)=\prod_{j=1}^{n}\left(1-u_{j} \bar{w}\right)^{-\left(\beta_{j}+2\right)}, \quad u \in U^{n} .
$$

Then $h_{w}$ is a bounded holomorphic function in $U^{n}$, so that $\mathcal{T}_{\beta} h_{w}=h_{w}$ by Theorem 2.2(i). Recalling $\Phi(z)=(z, \ldots, z)$, we thus have

$$
\mathcal{T}_{\beta} h_{w}(\Phi(z))=h_{w}(\Phi(z))=(1-z \bar{w})^{-(|\beta|+2 n)} .
$$

From (2.2) and (2.3), Fubini's theorem shows that for any $f \in A(U)$ and $z \in U$, 


$$
\begin{aligned}
\mathcal{D}_{\beta}\left(\mathcal{E}_{\beta} f\right)(z) & =(|\beta|+2 n-1) \int_{U}\left(1-|w|^{2}\right)^{|\beta|+2 n-2} \mathcal{T}_{\beta} h_{w}(\Phi(z)) f(w) d m_{1}(w) \\
& =(|\beta|+2 n-1) \int_{U} \frac{\left(1-|w|^{2}\right)^{|\beta|+2 n-2}}{(1-z \bar{w})^{|\beta|+2 n}} f(w) d m_{1}(w) \\
& =\mathcal{T}_{|\beta|+2 n-2} f(z)=f(z) .
\end{aligned}
$$

Theorem 1.1 is a direct consequence of Corollaries 2.3 and 2.4.

3. Extended Hardy inequalities. In order to prove the boundedness of integral operators in mixed norm spaces, we need to establish some useful inequalities concerning mixed integrals over $I^{n}$ or $I$, which are closely related to Hardy's inequalities when $n=1$ (see [HL], [Fl], [AB]).

Proposition 3.1. Let $b_{j}>a_{j}>0, c_{j}>0, \forall j=1, \ldots, n, \delta>0$, and let $g: I^{n} \rightarrow[0, \infty)$ be measurable. Assume either $0<k<1$ and $g$ is increasing in each variable, or $1 \leq k<\infty$. Then

$$
\begin{aligned}
& \int_{I}(1-\varrho)^{k|a|-1}\left(\int_{I^{n}} \frac{\prod_{j=1}^{n}\left(1-r_{j}\right)^{c_{j}-1}}{\prod_{j=1}^{n}\left(1-r_{j} \varrho\right)^{b_{j}}} g(r) d r\right)^{k} d \varrho \\
& \quad \leq K(a, b, c, k) \int_{I^{n}} \prod_{j=1}^{n}\left(1-r_{j}\right)^{k\left(a_{j}-b_{j}+c_{j}\right)-1} g^{k}(r) d r
\end{aligned}
$$

(ii)

$$
\begin{aligned}
\int_{I^{n}} \prod_{j=1}^{n}\left(1-r_{j}\right)^{k a_{j}-1} & \left(\int_{I^{n}} \frac{\prod_{j=1}^{n}\left(1-t_{j}\right)^{c_{j}-1}}{\prod_{j=1}^{n}\left(1-r_{j} t_{j}\right)^{b_{j}}} g(t) d t\right)^{k} d r \\
& \leq K(a, b, c, k) \int \prod_{I^{n}}^{n}\left(1-r_{j=1}\right)^{k\left(a_{j}-b_{j}+c_{j}\right)-1} g^{k}(r) d r
\end{aligned}
$$

$$
\begin{aligned}
\int_{I^{n}} \prod_{j=1}^{n}\left(1-r_{j}\right)^{k a_{j}-1}\left(\int_{I} \frac{(1-\varrho)^{\delta-1}}{\prod_{j=1}^{n}\left(1-r_{j} \varrho\right)^{b_{j}}} g(\varrho) d \varrho\right)^{k} d r \\
\quad \leq K(a, b, \delta, k) \int_{I}(1-\varrho)^{k(|a|-|b|+\delta)-1} g^{k}(\varrho) d \varrho .
\end{aligned}
$$

For the proof we need some technical lemmas.

Lemma 3.2. Let $b_{j}>a_{j}>0, j=1, \ldots, n$, and $r \in I^{n}$. Then

$$
\begin{gathered}
\int_{I} \frac{(1-\varrho)^{|a|-1}}{\prod_{j=1}^{n}\left(1-r_{j} \varrho\right)^{b_{j}}} d \varrho \leq K(a, b) \frac{1}{\prod_{j=1}^{n}\left(1-r_{j}\right)^{b_{j}-a_{j}}} ; \\
\int_{I^{n}} \frac{\prod_{j=1}^{n}\left(1-t_{j}\right)^{a_{j}-1}}{\prod_{j=1}^{n}\left(1-r_{j} t_{j}\right)^{b_{j}}} d t \leq K(a, b) \frac{1}{\prod_{j=1}^{n}\left(1-r_{j}\right)^{b_{j}-a_{j}}} .
\end{gathered}
$$


Proof. (i) When $n=1$, the inequality is well known (see for example $[\mathrm{SW}])$. We now apply induction on $n$ to deal with the general case. Assume that (i) holds for $n-1$. For any given $r=\left(r_{1}, \ldots, r_{n}\right) \in I^{n}$, let $r_{0}=\min \left\{r_{j}\right.$ : $j=1, \ldots, n\}$. Then

$$
\begin{aligned}
\int_{r_{0}}^{1} \frac{(1-\varrho)^{|a|-1}}{\prod_{j=1}^{n}\left(1-r_{j} \varrho\right)^{b_{j}}} d \varrho & \leq \frac{\left(1-r_{0}\right)^{a_{1}}}{\left(1-r_{0}^{2}\right)^{b_{1}}} \int_{r_{0}}^{1} \frac{(1-\varrho)^{a_{2}+\cdots+a_{n}-1}}{\prod_{j=2}^{n}\left(1-r_{j} \varrho\right)^{b_{j}}} d \varrho \\
& \leq \frac{1}{\left(1-r_{1}\right)^{b_{1}-a_{1}}} \frac{K}{\prod_{j=2}^{n}\left(1-r_{j}\right)^{b_{j}-a_{j}}} .
\end{aligned}
$$

Since $\prod_{j=1}^{n}\left(1-r_{j} \varrho\right)^{-b_{j}} \leq \prod_{j=1}^{n}(1-\varrho)^{-b_{j}}$ for any $\varrho \in[0,1)$, we also have

$$
\int_{0}^{r_{0}} \frac{(1-\varrho)^{|a|-1}}{\prod_{j=1}^{n}\left(1-r_{j} \varrho\right)^{b_{j}}} d \varrho \leq K \frac{1}{\left(1-r_{0}\right)^{|b|-|a|}} \leq K \frac{1}{\prod_{j=1}^{n}\left(1-r_{j}\right)^{b_{j}-a_{j}}} .
$$

This proves (i).

(ii) This is obvious since the integral can be decomposed as the product of integrals over $I$.

Applying the standard technique of Hardy-Littlewood [HL], we obtain the following inequality in the case of small indices.

Lemma 3.3. Let $0<p \leq 1$ and $b_{j} \geq 0, c_{j}>0, \forall j=1, \ldots, n$, and let $g: I^{n} \rightarrow[0, \infty)$ be increasing in each variable. Then there exists $K=K(p, b)$ such that

$$
\left\{\int_{I^{n}} \frac{\prod_{j=1}^{n}\left(1-t_{j}\right)^{c_{j}-1}}{\prod_{j=1}^{n}\left(1-r_{j} t_{j}\right)^{b_{j}}} g(t) d t\right\}^{p} \leq K \int_{I^{n}} \frac{\prod_{j=1}^{n}\left(1-t_{j}\right)^{p c_{j}-1}}{\prod_{j=1}^{n}\left(1-r_{j} t_{j}\right)^{p b_{j}}} g^{p}(t) d t .
$$

Proof. Let $\lambda_{k_{j}}=1-2^{-k_{j}}$ and $I_{k}=\prod_{j=1}^{n}\left[\lambda_{k_{j}-1}, \lambda_{k_{j}}\right)$. Then in $I_{k}$ we have

$$
\begin{gathered}
1-\lambda_{k_{j}} \leq 1-t_{j} \leq 2\left(1-\lambda_{k_{j}}\right), \quad 1-\varrho \lambda_{k_{j}} \leq 1-\varrho t_{j} \leq 2\left(1-\varrho \lambda_{k_{j}}\right), \\
g(t) \leq g\left(\lambda_{k}\right), \quad\left|I_{k}\right|=\prod_{j=1}^{n}\left(1-\lambda_{k_{j}}\right),
\end{gathered}
$$

where $\lambda_{k}=\left(\lambda_{k_{1}}, \ldots, \lambda_{k_{n}}\right), \varrho \in I$ and $t=\left(t_{1}, \ldots, t_{n}\right) \in I_{k}$.

We claim that

$$
\left\{\int_{I_{k}} \frac{\prod_{j=1}^{n}\left(1-t_{j}\right)^{c_{j}-1}}{\prod_{j=1}^{n}\left(1-r_{j} t_{j}\right)^{b_{j}}} g(t) d t\right\}^{p} \leq K \int_{I_{k+1}} \frac{\prod_{j=1}^{n}\left(1-t_{j}\right)^{p c_{j}-1}}{\prod_{j=1}^{n}\left(1-r_{j} t_{j}\right)^{p b_{j}}} g^{p}(t) d t .
$$

Indeed, the integrand on the left side of (3.2) is enlarged if we replace $t_{j}$ by the constant $\lambda_{k_{j}}$ and $g(t)$ by the constant $g\left(\lambda_{k}\right)$, up to a constant independent of $k$. Then we can calculate the resulting integral and its $p$ th power. The result is further enlarged if we replace $\lambda_{k}$ by $t \in I_{k+1}$, so (3.2) holds. 
Now we write the integral over $I^{n}$ as the sum of integrals over $I_{k}$, and then apply the inequality $(a+b)^{p} \leq a^{p}+b^{p}$ for $0<p<1$. The desired conclusion follows from (3.2) by summing over $k$.

Now we can prove the generalized Hardy inequality.

Proof of Proposition 3.1. (i) First assume that $1<k<\infty$. Set

$$
J=\int_{I^{n}} \frac{\prod_{j=1}^{n}\left(1-r_{j}\right)^{c_{j}-1}}{\prod_{j=1}^{n}\left(1-r_{j} \varrho\right)^{b_{j}}} g(r) d r .
$$

Rewrite the integrand of $J$ as the product of

$$
\prod_{j=1}^{n}\left(1-r_{j}\right)^{b_{j}-a_{j}-\varepsilon-1 / k^{\prime}} \prod_{j=1}^{n}\left(1-\varrho r_{j}\right)^{a_{j}-b_{j}}
$$

and

$$
\prod_{j=1}^{n}\left(1-r_{j}\right)^{c_{j}-b_{j}+a_{j}+\varepsilon-1 / k} \prod_{j=1}^{n}\left(1-\varrho r_{j}\right)^{-a_{j}} g(r) .
$$

Here $\varepsilon$ is a sufficiently small positive number and $k^{\prime}$ is the conjugate index of $k$. Applying Hölder's inequality and Lemma 3.2(ii), we have

$$
J^{k} \leq \frac{K}{\prod_{j=1}^{n}(1-\varrho)^{k \varepsilon}} \int_{I^{n}} \frac{\prod_{j=1}^{n}\left(1-r_{j}\right)^{k\left(c_{j}-b_{j}+a_{j}+\varepsilon\right)-1}}{\prod_{j=1}^{n}\left(1-\varrho r_{j}\right)^{k a_{j}}} g^{k}(r) d r .
$$

Note that here we used the assumption $b_{j}>a_{j}$.

For $0<k \leq 1$, Lemma 3.3 shows that

$$
J^{k} \leq K \int_{I^{n}} \frac{\prod_{j=1}^{n}\left(1-r_{j}\right)^{k c_{j}-1}}{\prod_{j=1}^{n}\left(1-\varrho r_{j}\right)^{k b_{j}}} g^{k}(r) d r .
$$

Consequently, for any $0<k<\infty, J^{k}$ can be estimated by the integral over $I^{n}$, so that Fubini's theorem and Lemma 3.2(i) yield the desired result.

(ii) Since the expression in brackets in (ii) is again $J$, and $J^{k}$ can be estimated by the integral over $I^{n}$, the desired result follows from Fubini's theorem.

(iii) Set

$$
\widetilde{J}=\int_{I} \frac{(1-\varrho)^{\delta-1}}{\prod_{j=1}^{n}\left(1-r_{j} \varrho\right)^{b_{j}}} g(\varrho) d \varrho .
$$

For $1<k<\infty$, we take $\varepsilon>0$ sufficiently small and rewrite the above integrand as the product of

$$
(1-\varrho)^{|b|-|a|-n \varepsilon-1 / k^{\prime}} \prod_{j=1}^{n}\left(1-r_{j} \varrho\right)^{a_{j}-b_{j}}
$$


and

$$
(1-\varrho)^{\delta-|b|+|a|+n \varepsilon-1 / k} \prod_{j=1}^{n}\left(1-r_{j} \varrho\right)^{-a_{j}} g(\varrho) .
$$

Then from Hölder's inequality and Lemma 3.2(i),

$$
\widetilde{J}^{k} \leq K \frac{1}{\prod_{j=1}^{n}\left(1-r_{j}\right)^{k \varepsilon}} \int_{I} \frac{(1-\varrho)^{k(\delta-|b|+|a|+n \varepsilon)-1}}{\prod_{j=1}^{n}\left(1-r_{j} \varrho\right)^{k a_{j}}} g^{k}(\varrho) d \varrho .
$$

If $0<k<1$, Lemma 3.3 shows that $\widetilde{J}^{k}$ can be estimated by the integral over $I$. The desired result now follows from Fubini's theorem and Lemma 3.2 (ii).

4. Weighted Bergman projections. In this section, we consider the boundedness of weighted Bergman operators on $L^{p, q, \alpha}\left(U^{n}\right)$. We refer to $[\mathrm{FR}],[\mathrm{SW}]$ for boundedness properties of weighted Bergman operators on $L^{p}$ spaces in the unit ball of $\mathbb{C}^{n}$.

THEOREM 4.1. Under Hypothesis 2.1,

(i) $\mathcal{T}_{\beta}: L^{p, q, \alpha}\left(U^{n}\right) \rightarrow H^{p, q, \alpha}\left(U^{n}\right)$ is bounded provided $1 \leq p<\infty$ and $1 \leq q \leq \infty$

(ii) $\mathcal{T}_{\beta}: H^{p, q, \alpha}\left(U^{n}\right) \rightarrow H^{p, q, \alpha}\left(U^{n}\right)$ is always bounded.

Let $\delta_{j k}$ be the Kronecker delta, i.e., $\delta_{j k}=1$ if $j=k$, and 0 otherwise.

Lemma 4.2. Let $u=\left(u_{1}, \ldots, u_{n}\right) \in U^{n}$ and $r_{j}=\left|u_{j}\right|, j=1, \ldots, n$. If $\alpha_{j}>1$, then for any $\varrho \in I$ and $t \in I^{n}$,

$$
\begin{aligned}
\int_{T} \frac{d \sigma_{1}(\zeta)}{\prod_{j=1}^{n}\left|1-\varrho \zeta \bar{u}_{j}\right|^{\alpha_{j}}} & \leq K(\alpha) \frac{1}{\prod_{j=1}^{n}\left(1-\varrho r_{j}\right)^{\alpha_{j}-\delta_{j, 1}}} ; \\
\int_{T^{n}} \frac{d \sigma_{n}(\zeta)}{\prod_{j=1}^{n}\left|1-t_{j} \zeta_{j} \bar{u}_{j}\right|^{\alpha_{j}}} & \leq K(\alpha) \frac{1}{\prod_{j=1}^{n}\left(1-t_{j} r_{j}\right)^{\alpha_{j}-1}} .
\end{aligned}
$$

Proof. The case of $n=1$ is well known (see [D]). Assertion (i) then follows from the inequality

$$
\prod_{j=2}^{n}\left|1-\varrho \zeta \bar{u}_{j}\right|^{-\alpha_{j}} \leq \prod_{j=2}^{n}\left(1-\varrho r_{j}\right)^{-\alpha_{j}} .
$$

Assertion (ii) is obvious since it can be reduced to the case $n=1$.

Proof of Theorem 4.1. For any function $F$ in $U^{n}$ and

$$
\beta_{j}>\frac{\alpha_{j}+1}{p}+\frac{1}{\min \{q, 1\}}-2, \quad \alpha_{j}>-1, \quad \forall j=1, \ldots, n,
$$


let

$$
G_{v}(u)=F(u) \prod_{j=1}^{n}\left(1-\bar{v}_{j} u_{j}\right)^{-\left(\beta_{j}+2\right)}
$$

for any $v, u \in U^{n}$. By (2.1) and the polar coordinates formula,

$$
\left|\mathcal{T}_{\beta} F(v)\right| \leq K \int_{I^{n}} \prod_{j=1}^{n}\left(1-r_{j}\right)^{\beta_{j}} M_{1}\left(r, G_{v}\right) d r .
$$

Assume that $1 \leq q \leq \infty$. Minkowski's inequality shows that

$$
M_{q}\left(t, \mathcal{T}_{\beta} F\right) \leq K \int_{I^{n}} \prod_{j=1}^{n}\left(1-r_{j}\right)^{\beta_{j}} M_{q}\left(t, M_{1}\left(r, G_{v}\right)\right) d r .
$$

Let $v, u \in U^{n}$ and $t_{j}=\left|v_{j}\right|, r_{j}=\left|u_{j}\right|$. We claim that

$$
M_{q}\left(t, M_{1}\left(r, G_{v}\right)\right) \leq K M_{q}(r, F) \prod_{j=1}^{n}\left(1-t_{j} r_{j}\right)^{-\left(\beta_{j}+1\right)} .
$$

From this claim together with (4.3), we find

$$
M_{q}\left(t, \mathcal{T}_{\beta} F\right) \leq K \int_{I^{n}} \frac{\prod_{j=1}^{n}\left(1-r_{j}\right)^{\beta_{j}}}{\prod_{j=1}^{n}\left(1-t_{j} r_{j}\right)^{\beta_{j}+1}} M_{q}(r, F) d r .
$$

Therefore, applying Proposition 3.1(ii) with $b_{j}=c_{j}=\beta_{j}+1, a_{j}=\left(\alpha_{j}+1\right) / p$, $k=p$ and $g(r)=M_{q}(r, F)$ we get

$$
\int_{I^{n}} \prod_{j=1}^{n}\left(1-t_{j}^{2}\right)^{\alpha_{j}} M_{q}^{p}\left(t, \mathcal{T}_{\beta} F\right) d t \leq K \int_{I^{n}} \prod_{j=1}^{n}\left(1-r_{j}^{2}\right)^{\alpha_{j}} M_{q}^{p}(r, F) d r .
$$

Here we used Hypothesis (2.1)(iii), which assures $b_{j}>a_{j}$.

To prove the case $1 \leq q \leq \infty$, it remains to prove claim (4.4). Rewrite $G_{v}(u)=G_{v}^{(1)}(u) G_{v}^{(2)}(u)$, where

$$
\begin{aligned}
& G_{v}^{(1)}(u)=F(u) \prod_{j=1}^{n}\left(1-\bar{v}_{j} u_{j}\right)^{-\left(\beta_{j}+2\right) / q}, \\
& G_{v}^{(2)}(u)=\prod_{j=1}^{n}\left(1-\bar{v}_{j} u_{j}\right)^{-\left(\beta_{j}+2\right) / q^{\prime}} .
\end{aligned}
$$

From Hölder's inequality we have

$$
\begin{aligned}
M_{1}\left(r, G_{v}\right) & \leq M_{q}\left(r, G_{v}^{(1)}\right) M_{q^{\prime}}\left(r, G_{v}^{(2)}\right) \\
& \leq K(q, \beta) M_{q}\left(r, G_{v}^{(1)}\right) \prod_{j=1}^{n}\left(1-t_{j} r_{j}\right)^{-\left(\beta_{j}+1\right) / q^{\prime}} .
\end{aligned}
$$


If $q=\infty$, then $G_{v}^{(1)}=F$ and $q^{\prime}=1$, so that (4.4) follows from (4.6). If $1 \leq q<\infty$, notice that by (1.1), the definition of $G_{v}^{(1)}$ and Lemma 4.2(ii),

$$
\begin{aligned}
M_{q}^{q}\left(t, M_{q}\left(r, G_{v}^{(1)}\right)\right) & =\int_{T^{n}} \int_{T^{n}}\left|G_{t \eta}^{(1)}(r \zeta)\right|^{q} d \sigma_{n}(\zeta) d \sigma_{n}(\eta) \\
& \leq K M_{q}^{q}(r, F) \prod_{j=1}^{n}\left(1-t_{j} r_{j}\right)^{-\left(\beta_{j}+1\right)},
\end{aligned}
$$

and the claim also follows from (4.6).

Assume now that $0<q<1$ and that $F$ is holomorphic in $U^{n}$. Then $G_{v}$ is holomorphic in $U^{n}$ for any given $v \in U^{n}$, so that $M_{1}\left(r, G_{v}\right)$ is increasing in each $r_{j}, j=1, \ldots, n$. Thus from (4.2) and Lemma 3.3, we obtain

$$
\left|\mathcal{T}_{\beta} F(v)\right|^{q} \leq K \int_{I^{n}} \prod_{j=1}^{n}\left(1-r_{j}\right)^{q\left(\beta_{j}+1\right)-1} M_{1}^{q}\left(r, G_{v}\right) d r .
$$

Combining this with

$$
M_{1}^{q}\left(r, G_{v}\right) \leq K(q) \prod_{j=1}^{n}\left(1-r_{j}\right)^{q-1} M_{q}^{q}\left(r, G_{v}\right),
$$

which holds since $G_{v}$ is holomorphic (see $[\mathrm{Fr}]$ ), we deduce that

$$
\left|\mathcal{T}_{\beta} F(v)\right|^{q} \leq K \int_{U^{n}} \frac{\prod_{j=1}^{n}\left(1-\left|u_{j}\right|^{2}\right)^{q\left(\beta_{j}+2\right)-2}}{\prod_{j=1}^{n}\left|1-v_{j} \bar{u}_{j}\right|^{q\left(\beta_{j}+2\right)}}|F(u)|^{q} d m_{n}(u) .
$$

Now, integrating over $T^{n}$ and changing the order of integration, from Lemma 4.2(ii) we have

$$
M_{q}^{q}\left(t, \mathcal{T}_{\beta} F\right) \leq K \int_{I^{n}} \frac{\prod_{j=1}^{n}\left(1-r_{j}\right)^{q\left(\beta_{j}+2\right)-2}}{\prod_{j=1}^{n}\left(1-t_{j} r_{j}\right)^{q\left(\beta_{j}+2\right)-1}} M_{q}^{q}(r, F) d r .
$$

By applying Proposition 3.1(ii), we obtain

$$
\int_{I^{n}} \prod_{j=1}^{n}\left(1-t_{j}^{2}\right)^{\alpha_{j}} M_{q}^{p}\left(t, \mathcal{T}_{\beta} F\right) d t \leq K \int_{I^{n}} \prod_{j=1}^{n}\left(1-r_{j}^{2}\right)^{\alpha_{j}} M_{q}^{p}(r, F) d r .
$$

This completes the proof.

5. Proof of Theorem 2.2. This section is devoted to proving Theorem 2.2 .

Proof of Theorem 2.2(i). By applying the method of [Sh2 (I), Proposition 2.3], every function in $L_{a}^{p, q, \alpha}\left(U^{n}\right)$ can be approximated by its slice functions, so $A\left(U^{n}\right)$ is dense in $H^{p, q, \alpha}\left(U^{n}\right)$. We claim that

$$
\left.\mathcal{T}_{\beta}\right|_{A\left(U^{n}\right)}=\mathrm{Id} \text {. }
$$


In fact the case $n=1$ is well known (see $[\mathrm{FR}]$ ) and the general case follows from this special case by iteration. More precisely, let $F \in A\left(U^{n}\right)$ and rewrite (2.1) as

$$
\begin{aligned}
\mathcal{T}_{\beta} F(v)= & \prod_{k=2}^{n}\left(\beta_{k}+1\right) \int_{U^{n-1}} \frac{\prod_{k=2}^{n}\left(1-\left|u_{k}\right|^{2}\right)^{\beta_{k}}}{\prod_{k=2}^{n}\left(1-v_{k} \bar{u}_{k}\right)^{\beta_{j}+2}} d m_{1}\left(u_{2}\right) \cdots d m_{1}\left(u_{n}\right) \\
& \cdot \int_{U}\left(\beta_{1}+1\right) \frac{\left(1-\left|u_{1}\right|^{2}\right)^{\beta_{1}}}{\left(1-v_{1} \bar{u}_{1}\right)^{\beta_{1}+2}} F\left(u_{1}, \ldots, u_{n}\right) d m_{1}\left(u_{1}\right) .
\end{aligned}
$$

Note that the second integral is equal to $F\left(v_{1}, u_{2}, \ldots, u_{n}\right)$. By continuing this procedure, we finally have

$$
\mathcal{T}_{\beta} F\left(v_{1}, \ldots, v_{n}\right)=F\left(v_{1}, \ldots, v_{n}\right),
$$

as desired. Thus the boundedness of $\mathcal{T}_{\beta}$ ensured by Theorem 4.1(ii) implies that

$$
\left.\mathcal{T}_{\beta}\right|_{H^{p, q, \alpha}\left(U^{n}\right)}=\mathrm{Id}
$$

Proof of Theorem 2.2(ii). Let $z \in U, u \in U^{n}$ and $\varrho=|z|, r_{j}=\left|u_{j}\right|$. Let

$$
G_{z}(u)=F(u) \prod_{j=1}^{n}\left(1-\bar{z} u_{j}\right)^{-\left(\beta_{j}+2\right)} .
$$

By (2.2) and the polar coordinates formula,

$$
\left|\mathcal{D}_{\beta} F(z)\right| \leq K \int_{I^{n}} \prod_{j=1}^{n}\left(1-r_{j}\right)^{\beta_{j}} M_{1}\left(r, G_{z}\right) d r .
$$

Assume that $0<q<1$ and that $F$ is holomorphic on $U^{n}$. Then $G_{z}$ is holomorphic on $U^{n}$ for any given $z \in U$. By Lemma 3.3,

$$
\left|\mathcal{D}_{\beta} F(z)\right|^{q} \leq K \int_{I^{n}} \prod_{j=1}^{n}\left(1-r_{j}\right)^{q\left(\beta_{j}+1\right)-1} M_{1}^{q}\left(r, G_{z}\right) d r .
$$

Since $M_{1}^{q}\left(r, G_{z}\right) \leq K(q) \prod_{j=1}^{n}\left(1-r_{j}\right)^{q-1} M_{q}^{q}\left(r, G_{z}\right)$ (see $\left.[\mathrm{Fr}]\right)$, we have

$$
\left|\mathcal{D}_{\beta} F(z)\right|^{q} \leq K \int_{U^{n}} \frac{\prod_{j=1}^{n}\left(1-\left|u_{j}\right|^{2}\right)^{q\left(\beta_{j}+2\right)-2}}{\prod_{j=1}^{n}\left|1-z \bar{u}_{j}\right|^{q\left(\beta_{j}+2\right)}}|F(u)|^{q} d m_{n}(u) .
$$

Consequently, Lemma 4.2(i) shows that

$$
M_{q}^{q}\left(\varrho, \mathcal{D}_{\beta} F\right) \leq K \int_{I^{n}} \frac{\prod_{j=1}^{n}\left(1-r_{j}\right)^{q\left(\beta_{j}+2\right)-2}}{\prod_{j=1}^{n}\left(1-\varrho r_{j}\right)^{q\left(\beta_{j}+2\right)-\delta_{j 1}}} M_{q}^{q}(r, F) d r .
$$

Applying Proposition 3.1(i), we obtain

$$
\int_{I}(1-\varrho)^{|\alpha|+(p / q+1)(n-1)} M_{q}^{p}\left(\varrho, \mathcal{D}_{\beta} F\right) d \varrho \leq K \int_{I^{n}} \prod_{j=1}^{n}\left(1-r_{j}\right)^{\alpha_{j}} M_{q}^{p}(r, F) d r .
$$

This proves the case $0<q<1$. 
Let $1 \leq q \leq \infty$. By (5.3), Minkowski's inequality shows that

$$
M_{q}\left(\varrho, \mathcal{D}_{\beta} F\right) \leq K \int_{I^{n}} \prod_{j=1}^{n}\left(1-r_{j}\right)^{\beta_{j}} M_{q}\left(\varrho, M_{1}\left(r, G_{z}\right)\right) d r .
$$

We claim that

$$
M_{q}\left(\varrho, M_{1}\left(r, G_{z}\right)\right) \leq K M_{q}(r, F) \prod_{j=1}^{n}\left(1-r_{j} \varrho\right)^{-\left(\beta_{j}+1+\left(1-\delta_{j 1}\right) / q\right)} .
$$

From (5.4), we have

$$
M_{q}\left(\varrho, \mathcal{D}_{\beta} F\right) \leq K \int_{I^{n}} \frac{\prod_{j=1}^{n}\left(1-r_{j}\right)^{\beta_{j}}}{\prod_{j=1}^{n}\left(1-\varrho r_{j}\right)^{\beta_{j}+1+\left(1-\delta_{j 1}\right) / q}} M_{q}(r, F) d r,
$$

and apply Proposition 3.1(i) to obtain the desired result.

It remains to prove (5.4). We rewrite $G_{z}(u)=G_{z}^{(1)}(u) G_{z}^{(2)}(u)$, where

$$
\begin{aligned}
& G_{z}^{(1)}(u)=F(u) \prod_{j=1}^{n}\left(1-\bar{z} u_{j}\right)^{-\left(\beta_{j}+2\right) / q}, \\
& G_{z}^{(2)}(u)=\prod_{j=1}^{n}\left(1-\bar{z} u_{j}\right)^{-\left(\beta_{j}+2\right) / q^{\prime}} .
\end{aligned}
$$

From Hölder's inequality and Lemma 4.2 we have

$$
\begin{aligned}
M_{1}\left(r, G_{z}\right) & \leq M_{q}\left(r, G_{z}^{(1)}\right) M_{q^{\prime}}\left(r, G_{z}^{(2)}\right) \\
& \leq K(q, \beta) M_{q}\left(r, G_{z}^{(1)}\right) \prod_{j=1}^{n}\left(1-|z| r_{j}\right)^{-\left(\beta_{j}+1\right) / q^{\prime}} .
\end{aligned}
$$

If $q=\infty$, then $G_{z}^{(1)}=F$ and $q^{\prime}=1$, so that claim (5.4) follows from (5.6). If $1 \leq q<\infty$, notice that

$$
\begin{aligned}
M_{q}^{q}\left(\varrho, M_{q}\left(r, G_{z}^{(1)}\right)\right) & =\iint_{T T^{n}}\left|G_{\varrho \eta}^{(1)}(r \zeta)\right|^{q} d \sigma_{n}(\zeta) d \sigma_{1}(\eta) \\
& \leq K M_{q}^{q}(r, F) \prod_{j=1}^{n}\left(1-r_{j} \varrho\right)^{-\left(\beta_{j}+2-\delta_{j 1}\right)} .
\end{aligned}
$$

Hence claim (5.4) also follows from (5.6). This ends the proof.

Proof of Theorem 2.2(iii). Let $f$ be holomorphic on $U$ and let

$$
G_{u}(z)=f(z) \prod_{j=1}^{n}\left(1-z \bar{u}_{j}\right)^{-\left(\beta_{j}+2\right)}
$$

for any $u \in U^{n}$ and $z \in U$. From (2.3),

$$
\left|\mathcal{E}_{\beta} f(u)\right| \leq K \int_{I}(1-\varrho)^{|\beta|+2 n-2} M_{1}\left(\varrho, G_{u}\right) d \varrho .
$$


First assume that $0<q<1$. Since $G_{u}$ is holomorphic on $U$ for any given $u \in U^{n}$, it follows from Lemma 3.3 with $n=1$ that

$$
\left|\mathcal{E}_{\beta} f(u)\right|^{q} \leq K \int_{I}(1-\varrho)^{q(|\beta|+2 n-1)-1} M_{1}^{q}\left(\varrho, G_{u}\right) d \varrho .
$$

Notice that $M_{1}^{q}\left(\varrho, G_{u}\right) \leq K(q)(1-\varrho)^{q-1} M_{q}^{q}\left(\varrho, G_{u}\right)$. Recalling the definition of $G_{u}$ in (5.7) we have

$$
\left|\mathcal{E}_{\beta} f(u)\right|^{q} \leq K \int_{U} \frac{\left(1-|z|^{2}\right)^{q(|\beta|+2 n)-2}}{\prod_{j=1}^{n}\left|1-z \bar{u}_{j}\right|^{q\left(\beta_{j}+2\right)}}|f(z)|^{q} d m_{1}(z),
$$

so that Lemma 4.2(ii) gives

$$
M_{q}^{q}\left(r, \mathcal{E}_{\beta} f\right) \leq K \int_{I} \frac{(1-\varrho)^{q(|\beta|+2 n)-2}}{\prod_{j=1}^{n}\left(1-\varrho r_{j}\right)^{q\left(\beta_{j}+2\right)-1}} M_{q}^{q}(\varrho, f) d \varrho,
$$

and Proposition 3.1(iii) implies

$$
\int_{I^{n}} \prod_{j=1}^{n}\left(1-r_{j}\right)^{\alpha_{j}} M_{q}^{p}\left(r, \mathcal{E}_{\beta} f\right) d r \leq K \int_{I}(1-\varrho)^{|\alpha|+(p / q+1)(n-1)} M_{q}^{p}(\varrho, f) d \varrho .
$$

Now we assume that $1 \leq q \leq \infty$. Then, from (5.8), Minkowski's inequality shows that

$$
M_{q}\left(r, \mathcal{E}_{\beta} f\right) \leq K \int_{I}(1-\varrho)^{|\beta|+2 n-2} M_{q}\left(r, M_{1}\left(\varrho, G_{u}\right)\right) d \varrho .
$$

We claim that, for $r \in I^{n}$ defined by $r_{j}=\left|u_{j}\right|$ and $\varrho=|z| \in I$,

$$
M_{q}\left(r, M_{1}\left(\varrho, G_{u}\right)\right) \leq K M_{q}(\varrho, f) \prod_{j=1}^{n}\left(1-r_{j} \varrho\right)^{-\left(\beta_{j}+1+\left(1-\delta_{j 1}\right) / q^{\prime}\right)} .
$$

From this claim and (5.9), we find

$$
M_{q}\left(r, \mathcal{E}_{\beta} f\right) \leq K \int_{I} \frac{(1-\varrho)^{|\beta|+2 n-2}}{\prod_{j=1}^{n}\left(1-\varrho r_{j}\right)^{\beta_{j}+1+\left(1-\delta_{j 1}\right) / q^{\prime}}} M_{q}(\varrho, f) d \varrho .
$$

Therefore, applying Proposition 3.1(iii) we get the desired result:

$$
\int_{I^{n}} \prod_{j=1}^{n}\left(1-r_{j}\right)^{\alpha_{j}} M_{q}^{p}\left(r, \mathcal{E}_{\beta} f\right) d r \leq K \int_{I}(1-\varrho)^{|\alpha|+(p / q+1)(n-1)} M_{q}^{p}(\varrho, f) d \varrho .
$$

It remains to prove the claim in (5.10). To this end, write $G_{u}(z)=$ $G_{u}^{(1)}(z) G_{u}^{(2)}(z)$, where

$$
\begin{aligned}
& G_{u}^{(1)}(z)=f(z) \prod_{j=1}^{n}\left(1-z \bar{u}_{j}\right)^{-\left(\beta_{j}+2\right) / q} \\
& G_{u}^{(2)}(z)=\prod_{j=1}^{n}\left(1-z \bar{u}_{j}\right)^{-\left(\beta_{j}+2\right) / q^{\prime}}
\end{aligned}
$$


From Hölder's inequality and Lemma 4.2(i) we have, for any $\beta_{j}>-1$,

$$
\begin{aligned}
M_{1}\left(\varrho, G_{u}\right) & \leq M_{q}\left(\varrho, G_{u}^{(1)}\right) M_{q^{\prime}}\left(\varrho, G_{u}^{(2)}\right) \\
& \leq K(q, \beta) M_{q}\left(\varrho, G_{u}^{(1)}\right) \prod_{j=1}^{n}\left(1-\left|u_{j}\right| \varrho\right)^{-\left(\beta_{j}+2-\delta_{j 1}\right) / q^{\prime}} .
\end{aligned}
$$

If $q=\infty$, then $G_{u}^{(1)}=f$ and $q^{\prime}=1$ so that (5.10) follows directly from (5.12). If $1 \leq q<\infty$, notice that in virtue of (1.1), Fubini's theorem and Lemma 4.2 ,

$$
\begin{aligned}
M_{q}^{q}\left(r, M_{q}\left(\varrho, G_{u}^{(1)}\right)\right) & =\int_{T^{n}} \int_{T}\left|G_{r \eta}^{(1)}(\varrho \zeta)\right|^{q} d \sigma_{1}(\zeta) d \sigma_{n}(\eta) \\
& \leq K M_{q}^{q}(\varrho, f) \prod_{j=1}^{n}\left(1-r_{j} \varrho\right)^{-\left(\beta_{j}+1\right)}
\end{aligned}
$$

Then (5.10) follows from (5.12) and Lemma 3.2(i). This completes the proof.

\section{References}

[AB] P. Ahern and J. Bruna, Maximal and area integral characterizations of HardySobolev spaces in the unit ball of $\mathbb{C}^{n}$, Rev. Mat. Iberoamericana 4 (1988), 123-153.

[AJ] P. Ahern and M. Jevtić, Duality and multipliers for mixed norm spaces, Michigan Math. J. 30 (1983), 53-64.

[BP] A. Benedek and R. Panzone, The space $L^{P}$, with mixed norm, Duke Math. J. 28 (1961), 301-324.

$[\mathrm{CM}] \quad$ C. C. Cowen and B. D. MacCluer, Composition Operators on Spaces of Analytic Functions, CRC Press, Boca Raton, FL, 1995.

[Det] J. Detraz, Restriction à la diagonale des classes de Hardy du bidisque, C. R. Acad. Sci. Paris 287 (1978), 997-999.

[DjS] A. E. Djrbashian and F. A. Shamoian, Topics in the Theory of $A_{\alpha}^{p}$ Spaces, Leipzig, Teubner, 1988.

[D] P. L. Duren, Theory of $H^{p}$ Spaces, Academic Press, New York, 1970.

[DS] P. L. Duren and A. L. Shields, Restrictions of $H^{p}$ functions to the diagonal of the polydisc, Duke Math. J. 42 (1975), 751-753.

[Fl] T. M. Flett, The dual of an inequality of Hardy and Littlewood and some related inequalities, J. Math. Anal. Appl. 38 (1972), 746-765.

[FR] F. Forelli and W. Rudin, Projections on spaces of holomorphic functions in the ball, Indiana Univ. Math. J. 24 (1974), 593-602.

[Fr] A. P. Frazier, The dual space of $H^{p}$ of the polydisc for $0<p<1$, Duke Math. J. 39 (1972), 369-379.

[HL] G. H. Hardy and J. E. Littlewood, Some properties of functional integrals II, Math. Z. 34 (1932), 403-439.

[HLP] G. H. Hardy, J. E. Littlewood and G. Pólya, Inequalities, Cambridge Univ. Press, 1934.

[HO] C. Horowitz and D. Oberlin, Restriction of $H^{p}$ functions to the diagonal of $U^{n}$, Indiana Univ. Math. J. 24 (1975), 767-772. 
[J] M. Jevtić, Projection theorems, fractional derivatives and inclusion theorems for mixed-norm spaces on the ball, Analysis 9 (1989), 83-105.

[L] D. H. Luecking, Embedding theorems for spaces of analytic functions via Khinchine's inequality, Michigan Math. J. 40 (1993), 333-358.

[MR] B. Moulin et J. P. Rosay, Sur la restriction des fonctions pluri-sous-harmoniques à la diagonale du polydisque, Indiana Univ. Math. J. 26 (1977), 869-973.

[Pa] M. Pavlović, Decompositions of $L^{p}$ and Hardy spaces of polyharmonic functions, J. Math. Anal. Appl. 216 (1997), 499-509.

[RL] G. B. Ren and C. W. Liu, Diagonal mappings on bounded symmetric domains, Chinese J. Contemp. Math. 22 (2001), 11-22.

[Ru1] W. Rudin, Function Theory in Polydiscs, Benjamin, New York, 1969.

[Ru2] - Function Theory in the Unit Ball of $\mathbb{C}^{n}$, Springer, Berlin, 1980.

[Sh] F. A. Shamoian, Diagonal mapping and problems of representation in anisotropic spaces of functions that are holomorphic in a polydisc, Siberian Math. J. 31 (1990), 350-365.

[Sha] J. H. Shapiro, Mackey topologies, reproducing kernels, and diagonal maps on the Hardy and Bergman spaces, Duke Math. J. 43 (1976), 187-202.

[Sh1] J. H. Shi, Inequalities for the integral means of holomorphic functions and their derivatives in the ball of $\mathbb{C}^{n}$, Trans. Amer. Math. Soc. 328 (1991), 619-637.

[Sh2] -, Duality and multipliers for mixed norm spaces in the ball (I), (II), Complex Variables 25 (1994), 119-130; 131-157.

[SR] J. H. Shi and G. B. Ren, Boundedness of the Cesàro operator on mixed norm spaces, Proc. Amer. Math. Soc. 126 (1998), 3553-3560.

[SW] A. L. Shields and D. L. Williams, Bounded projections, duality and multipliers in spaces of harmonic functions, J. Reine Angew. Math. 299/300 (1978), 256-279.

Department of Mathematics

University of Science and Technology of China

Hefei, Anhui 230026, P.R. China

E-mail: rengb@ustc.edu.cn

shijh@ustc.edu.cn
Current address of G. B. Ren:

Department of Mathematics

University of Aveiro 3810-193 Aveiro, Portugal

E-mail: ren@mat.ua.pt

Received August 13, 2002

Revised version December 4, 2003 